\title{
Hypertension in elderly
}

\section{Ali Oto}

${ }^{1)}$ MD, FESC, FACC, Hacettepe Univ of Medical School, Ankara, Turkey

With increase in modern healthcare access thanks to the developments in technology, the advances in preventive and treating medicine and the improvements in health organization, the expected length of life is prolonged, and the population ages rapidly. The elderly people $\geq 60$ years is the most rapidly growing segment in the population. In our country, the observation of increase in the aging population, was clearly revealed with the last two population censuses. In the last census, population $\geq 75$ years nearly doubled compared to the previous census.

With the aging, both the prevalence and incidence of the cardiovascular events mainly stroke and myocardial infarction increase. Cardiac failure is a highly important problem in elderly that we face, and its incidence increases every passing day. Overall difficulty in blood pressure control in the society, is more significant in the elderly population, and the already low blood pressure control rates decrease to very low levels in elderly.

Isolated systolic hypertension ( $>140 \mathrm{mmHg}$ systolic and $>90 \mathrm{mmHg}$ diastolic blood pressure values) is the most frequent form of high blood pressure seen in el- derly. The frequency of high blood pressure increases with age, and isolated systolic hypertension is observed in $2 / 3$ of the people over 60 years old and in $3 / 4$ of the people over 75 years old. Isolated systolic hypertension is the most important parameter in elderly with regards to cardiovascular risk; thus the main blood pressure treatment target is the isolated systolic hypertension.

Progressive stiffness in the aortic wall emerging with aging, decrease in the balancing effect of aorta on flow volume, progressive increase in the systolic blood pressure as a result of these, and decrease in diastolic blood pressure, togethwer with the widened pulse pressure constitute the key pathophysiological steps of hypertension in elderly. In addition to this, impairment of renal functions, sodium retention, impaired baroreceptor function, comorbidities, multiple drug use and the difficulties coming with advancing age, are the important aspects making the antihypertensive therapy difficult in this age group. Also the decrease in diastolic blood pressure, is a condition that may impair coronary circulation perfused at dilation.

Secondary hypertension due to renal artery stenosis 
should always be kept in mind particularly in elderly. In general, stage 2 hypertension with recent onset, hypokalemia without diuretic treatment of resistant hypokalemia under diuretic treatment, progressive creatinine elevation under proper antihypertensive treatment and hypertension resistant to triple medication treatment should direct physicians to secondary hypertension, and further investigation should be performed.

While there is a great body of evidence regarding the beneficial effects of antihypertensive treatment in elderly patients, there is conflicting information on the effectf of antihypertensive therapy in highly advanced age (>80). The most important reasons for this are that there is no study targeting this age group, the data is mostly based on subgroup analyses, and exclusion of advanced age group in many trials. Therefore, various recently published guidelines cannot provide a clear recommendation. Interestingly, the meta-analyses performed on this subject gave confusing results.

For example, in INDANA meta-analysis including 1670 patients, while $36 \%$ decrease is observed in the risk of stroke with antihypertensive treatment, mortality was reported to increase by $14 \%$. When HYVET Pilot study gave a similar result, conducting a placebocontrolled, prospective trial became an ethical issue, and the beneficial results obtained with the indapamid and perindopril combination in PROGRESS trial led authors to conduct HYVET trial by using these two drugs. Conducted as an international, multicenter, randomized, double-blind and placebo-controlled trial, HYVET Trial included nearly 5000 patients over 80 years old and having 160-199 $\mathrm{mmHg}$ of systolic and $>110 \mathrm{mmHg}$ diastolic blood pressure value. First $2 \mathrm{mg}$ and then $4 \mathrm{mg}$ perindopril was added to $1.5 \mathrm{mg}$ SR indapamid versus placebo, and the target blood pressure value was determined as $150-80 \mathrm{mmHg}$. At the end of a mean follow-up of nearly 2 years, in the stroke treatment group, $30 \%$ decrease in the risk of stroke was observed, and more importantly $21 \%$ decrease was obtained in all deaths. $64 \%$ decrease obtained in cardiac failure is highly dramatic.
Results coming from the subgroup analyses and the recently published meta-analysis results show that antihypertensive therapy in elderly have a beneficial effect in preventing the development of dementia. And this is an important clue supporting the benefit of antihypertensive therapy in elderly.

While there is no consensus on the first choice of treatment in older hypertensives, there is a recent tendency towards avoiding beta blockers, and preferring diuretics or calcium channel blockers. Blood pressure control in older hypertensive mostly requires 2-3 drugs. Fixed dose combinations may be appropriate because of their ease of use, and low side effects due to lowdose components.

In elderly patient, antihypertensive drug should be initiated at its low-dose, the dose should be increased with follow-ups in close intervals, however orthostatic hypotension and extreme decrease in diastolic blood pressure (>60 mmHg) should be kept in mind. During the antihypertensive drug choice process, comorbidities (COPD, BPH, DM, renal dysfunctions, etc.) and drug interactions should be considered. However, it should be remembered that nobody is too old to start antihypertensive treatment.

\section{Important Notes}

1. Hypertension is a serious problem seen $3 / 4$ of people over 60 years and older.

2. With the aging population, advanced age group segment rapidly grows; in fact in our day, it is the fastest growing part of the society.

3. Cardiovascular risk due to hypertension in elderly is related with elevation of systolic blood pressure, widening of pulse pressure and decrease in diastolic blood pressure. In this context, systolic blood pressure constitutes the key target for treatment.

4. Antihypertensive medication therapy in elderly has various characteristics. 